\title{
Differential carrier lifetime in oxide-confined vertical cavity lasers obtained from electrical impedance measurements
}

\author{
G. E. Giudice, ${ }^{\text {a) }}$ D. V. Kuksenkov, and H. Temkin \\ Electrical Engineering Department, Texas Tech University, Lubbock, Texas 79409 \\ K. L. Lear ${ }^{\text {b) }}$ \\ MicroOptical Devices, Inc., Albuquerque, New Mexico 87109
}

(Received 10 July 1998; accepted for publication 14 December 1998)

\begin{abstract}
Differential carrier lifetime measurements were performed on index-guided oxide-confined vertical cavity surface emitting lasers operating at $980 \mathrm{~nm}$. Lifetimes were extracted from laser impedance measurements at subthreshold currents, with device size as a parameter, using a simple small-signal model. The carrier lifetimes ranged from $21 \mathrm{~ns}$ at $9 \mu \mathrm{A}$, to about $1 \mathrm{~ns}$ at a bias close to threshold. For a $6 \times 6 \mu \mathrm{m}^{2}$ oxide aperture device the threshold carrier density was $n_{\text {th }} \sim 2 \times 10^{18} \mathrm{~cm}^{-3}$. The effect of carrier diffusion was also considered. An ambipolar diffusion coefficient of $D$ $\sim 11 \mathrm{~cm}^{2} \mathrm{~s}^{-1}$ was obtained. (C) 1999 American Institute of Physics. [S0003-6951(99)00507-0]
\end{abstract}

Carrier lifetime measurements are an important tool in semiconductor laser characterization. ${ }^{1,2}$ Recently, a new electrical technique for differential carrier lifetime measurements was developed. ${ }^{2}$ It originally applied to edge emitting lasers with bulk active region. More recently, it has been applied to vertical cavity surface emitting lasers (VCSELs) ${ }^{3}$ with quantum wells (QWs) in the active region. In this letter, experimental results on differential carrier lifetimes in indexguided oxide-confined VCSELs are reported. Lifetimes were obtained from laser impedance measurements at subthreshold currents and the equivalent circuit modeling. Threshold carrier densities and the corresponding recombination parameters were calculated as a function of device size. In addition, the effect of lateral carrier diffusion out of the active region was also considered.

Devices studied in this work were oxide-confined InGaAs VCSELs lasing at $980 \mathrm{~nm}$. The current aperture was obtained by partial oxidation of two quarter-wavelength GaAlAs layers. ${ }^{4}$ Lasers with aperture sizes in the range of 6-12 $\mu \mathrm{m}$ had room temperature threshold currents from 220 to $600 \mu \mathrm{A}$. The laser impedance was measured from $1 \mathrm{MHz}$ up to $3 \mathrm{GHz}$. Measurements were performed for bias currents between $9 \mu \mathrm{A}$ and threshold. Figure 1 presents the frequency dependence of the measured impedance of a device with a $\sim 8 \times 8 \mu \mathrm{m}^{2}$ active region, with a threshold current $I_{\text {th }}$ $\approx 240 \mu \mathrm{A}$, at $40 \mu \mathrm{A}$ of drive current. The measured laser impedance was modeled using the small-signal subthreshold equivalent circuit shown in the inset of Fig. 1. The parameters included in this model were the following: an inductance $L$ associated with a bond wire, a parallel combination of a resistance $R_{m}$ and capacitance $C_{m}$ associated with the top mirror interfaces, and a parallel combination of a resistance $R_{d}$ and a capacitance $C_{a}$ representing the active region. The impedance of the bottom mirror was neglected. The total impedance of the circuit can be written as

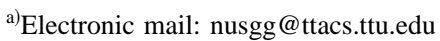

${ }^{b)}$ Also with Sandia National Laboratories, Albuquerque, NM 55448.
}

$$
\begin{aligned}
Z_{\mathrm{eq}}(\omega)= & \frac{R_{m}}{1+\left(R_{m} C_{m} \omega\right)^{2}}+\frac{R_{d}}{1+\left(R_{d} C_{a} \omega\right)^{2}} \\
& +j \omega\left(L-\frac{R_{m}^{2} C_{m}}{1+\left(R_{m} C_{m} \omega\right)^{2}}-\frac{R_{d} \tau_{d}}{1+\left(R_{d} C_{a} \omega\right)^{2}}\right),
\end{aligned}
$$

where $\tau_{d}=R_{d} C_{a}$ is the characteristic time equal to the differential carrier lifetime. ${ }^{5,6}$ This model does not consider the carrier transport and capture dynamics discussed elsewhere ${ }^{7,8}$ because it was assumed that the capture time is small and the escape time from the wells is very large compared to the carrier lifetime in the wells. In this simple model, $\tau_{d}$ is the effective lifetime of the carriers confined in the quantum wells, without considering the carrier distribution in the barriers and separate confinement regions.

Bias dependence of circuit parameters was studied as a function of device size. The differential carrier lifetimes were extracted by fitting the real and imaginary parts of the

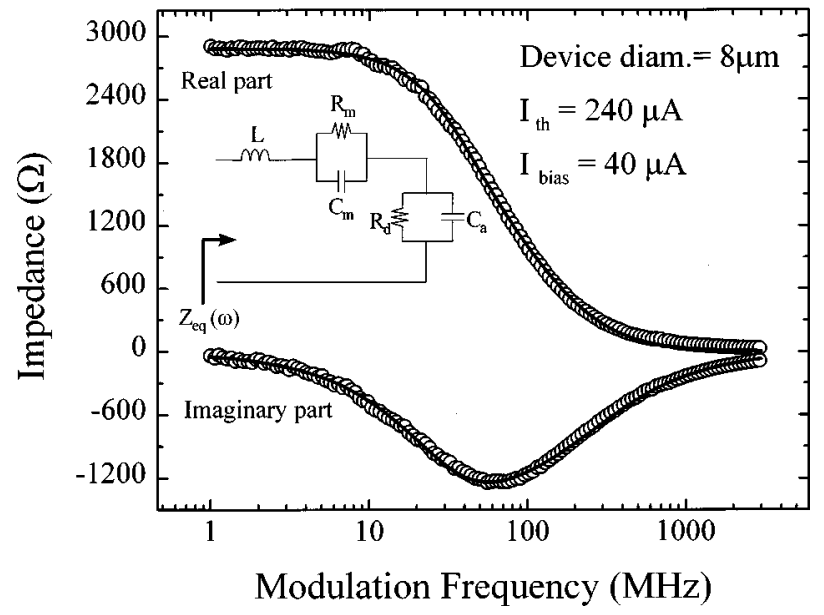

FIG. 1. Measured and fitted impedance of a 8 - $\mu$ m-diam VCSEL $\left(I_{\text {th }}\right.$ $=240 \mu \mathrm{A})$ vs frequency at $I=40 \mu \mathrm{A}$. Points are experimental data and lines are the best fit using the real and imaginary parts of Eq. (1). The inset shows the small-signal subthreshold VCSEL equivalent circuit. 


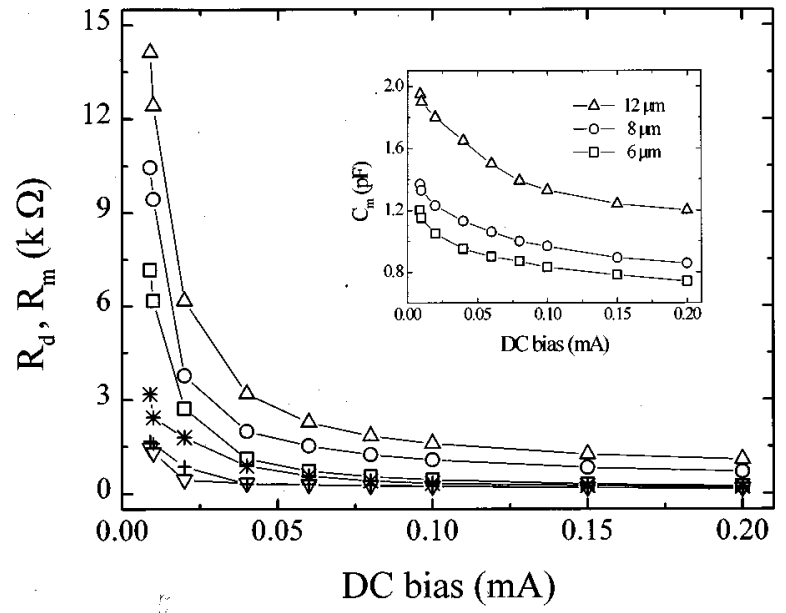

FIG. 2. Differential resistance values for 6 (up triangles), 8 (circles), and 12 $\mu \mathrm{m}$ (squares) device diameter as a function of bias current. Mirror resistance values for 6 (down triangles), 8 (crosses), and $12 \mu \mathrm{m}$ (stars) device diameter as a function of bias current. In the inset is displayed the mirror capacitance for the same device diameters as a function of bias current. Lines are drawn as a visual aid.

measured laser impedance with the equivalent circuit [Eq. (1)], using a calculated bond wire inductance $L \sim 0.6 \mathrm{nH}$, and considering $R_{d}, C_{a}, R_{m}$, and $C_{m}$ as fitting parameters. The results of the fitting are also displayed in Fig. 1 (solid line). The fit is in good agreement with the measured laser impedance yielding $\tau_{d} \sim 3.5 \mathrm{~ns}, \quad R_{d} \sim 2.0 \mathrm{k} \Omega, \quad C_{a} \sim 1.8 \mathrm{pF}, \quad R_{m}$ $\sim 0.8 \mathrm{k} \Omega, C_{m} \sim 1.0 \mathrm{pF}$, for a device with $\sim 8 \mu \mathrm{m}$ oxide aperture, at $40 \mu \mathrm{A}$ of bias current. The magnitudes of $R_{d}$ and $R_{m}$ were also measured as a function of the bias current under static conditions with very close agreement to the dynamic measurements. Figure 2 shows the variation of the differential resistance $R_{d}$ and the mirror resistance $R_{m}$ versus the injected current. The differential resistance $R_{d}$ in all samples was inversely proportional to the drive current, as is expected from the diode equation. The inset of Fig. 2 also shows variation of $C_{m}$ versus bias current, with the device size as a parameter. The resistance $R_{m}$ and capacitance $C_{m}$, associated with the top Bragg mirror, are also increasing at very low bias. The origin of this effect is not clearly understood. It could be due to a nonlinear impedance associated with band offsets at the Bragg mirror heterointerfaces.

The carrier density $n$ and the drive current $I$ are related by the rate equation ${ }^{9}$

$$
\frac{\partial n}{\partial t}=D \nabla^{2} n+\frac{I}{e V_{a}}-R(n),
$$

where $D$ is the diffusion coefficient, $e$ is the electron charge, $V_{a}$ is the active region volume, and $R(n)$ is the total recombination rate. In the steady state, $\partial n / \partial t=0$, and assuming that the carrier density does not vary significantly over the active region dimensions, the diffusion term in Eq. (2) is neglected. The recombination rate $R(n)$, without stimulated emission, is usually related to the carrier concentration $n$ through the expression $R(n)=A n+B n^{2}+C n^{3}$, where the first term is attributed to nonradiative recombination such as trap and surface recombination, the second term is the sponDownloaded 29 Oct 2004 to 129.82.229.173. Redistribution subject taneous emission rate, and the third term describes the Auger recombination. ${ }^{9}$ The differential carrier lifetime is defined as the derivative of the recombination rate

$$
\frac{1}{\tau_{d}(n)}=\frac{\partial R(n)}{\partial n}=A+2 B n+3 C n^{2} .
$$

The recombination coefficients can now be evaluated considering that the injected current is related to the carrier density by

$$
I(n)=\frac{e V_{a} R(n)}{k}=\frac{e V_{a}}{k}\left(A n+B n^{2}+C n^{3}\right),
$$

where $k$ is the injection efficiency, and $A, B$, and $C$ are the nonradiative, radiative, and Auger recombination coefficients, respectively.

Depending on the device size, lateral diffusion of carriers out of the active region could be an important mechanism limiting the laser performance. For each device size, the effect of lateral diffusion of carriers was studied by first determining the lifetime as a function of the drive current. Threshold carrier density and recombination parameters were then determined by defining an effective volume of the active region $V_{\text {eff }}$. The effective volume consists of the real device active volume $V_{a}$ and the diffused region. Thus, to obtain the recombination coefficients and the threshold carrier density from the measured differential lifetime, the injected current and the total recombination rate were related by

$$
I(n)=\frac{e V_{\mathrm{eff}}}{k}\left(A n+B n^{2}+C n^{3}\right),
$$

where $V_{\text {eff }}$ is the effective active region volume defined as $V_{\text {eff }} \approx N_{w} L_{w} r_{\text {eff }}^{2}$. Here, $N_{w}$ is the number of QWs in the active region, $L_{w}$ is the thickness of the QWs, and $r_{\text {eff }}$ is an equivalent radius approximated by $r_{\text {eff }} \approx 2 \sqrt{D \tau_{d}}+r_{o}$, where $r_{o}$ is the nominal active region radius without carrier diffusion. The threshold carrier density and recombination parameters were calculated taking $k=0.9,{ }^{10}$ and fitting the differential carrier lifetime as a function of injected current with both expressions, Eqs. (3) and (5). Using the same approach for each device size, the fitting procedure yielded threshold densities of $n_{\mathrm{th}} \sim 1.70 \times 10^{18} \mathrm{~cm}^{-3}$ for both 12 and $8 \mu \mathrm{m}$ and $1.95 \times 10^{18} \mathrm{~cm}^{-3}$ for $6 \mu \mathrm{m}$ device diameters. Differential lifetimes obtained from impedance measurements are plotted as a function of the bias current, with the device size as a parameter, in Fig. 3. The lifetimes obtained for oxide defined lasers are larger than the lifetimes obtained previously for GaAs gain guided QW VCSELs, with similar active volumes. ${ }^{3}$ To further confirm the data, and to check the validity of the proposed equivalent circuit, carrier lifetimes were also measured using the traditional small-signal optical response technique. ${ }^{1}$ The optical response was obtained with an optical spectrum analyzer and a fast photodetector with a flat response from $1 \mathrm{MHz}$ to $1 \mathrm{GHz}$. The measured response was corrected for the laser impedance, to account for the frequency dependent modulation amplitude, and fitted with a single-pole response function. The results are shown in the inset of Fig. 3. Good agreement was obtained between the lifetime values measured by the electrical and optical techniques. For each of the devices studied in this work, the to AlP license or copyright, see http://apl.aip.org/apl/copyright.jsp 


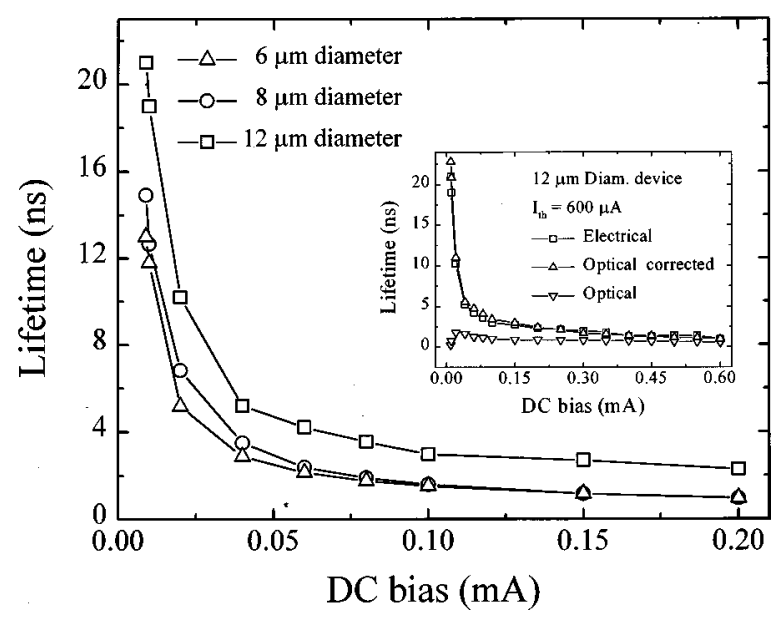

FIG. 3. Differential carrier lifetimes for 6 (triangles), 8 (circles), and $12 \mu \mathrm{m}$ (squares) device diameter as a function of bias current. The inset shows differential carrier lifetimes obtained using the electrical (squares), impedance corrected optical (up triangles), and traditional uncorrected optical (down triangles) techniques for a $12 \mu \mathrm{m}$ device diameter. Lines are drawn as a visual aid.

threshold carrier density value, recombination parameters, and diffusion coefficient were obtained from the fit to lifetime dependence on the drive current.

It was possible to obtain a good fit to the experimental $\tau_{d}(I)$ characteristics using the same values of $A, B, C$, and $D$ coefficients for all the device sizes. The parameter $A$, usually associated with nonradiative recombination, was considerably smaller $\left(A \sim 1 \times 10^{5} \mathrm{~s}^{-1}\right)$ than the values found previously in gain guided VCSELs $\left(A \sim 0.5-2.5 \times 10^{8} \mathrm{~s}^{-1}\right) .^{5}$ This suggests that the nonradiative, defect related, recombination processes are negligible in our lasers. The radiative recombination coefficient estimated here, $B \sim 1.3 \times 10^{-10} \mathrm{~cm}^{3} \mathrm{~s}^{-1}$, is the same as the value recently reported in $\mathrm{In}_{0.35} \mathrm{Ga}_{0.65} \mathrm{As} / \mathrm{GaAs}$ edge emitting multiple QW lasers. ${ }^{11}$ The values of Auger recombination coefficients found in the literature present noticeable differences depending on the particular method of measurement and the material system. In general, the values reported for InGaAs QWs are higher than those for GaAs and AlGaAs QWs. ${ }^{12}$ The Auger coefficient $C \sim 4 \times 10^{-29} \mathrm{~cm}^{6} \mathrm{~s}^{-1}$ estimated for our devices, for all threshold carrier densities extracted here, is approximately one order of magnitude higher than the value obtained previously for GaAs QW VCSELs. ${ }^{3}$

The best fit of the experimental data was obtained with a diffusion coefficient $D \sim 11 \mathrm{~cm}^{2} \mathrm{~s}^{-1}$. This result was independent of the device size. The diffusion of carriers out of the active region is commonly considered in the rate equations, defining a surface recombination velocity $S_{\text {eff }}$ of magnitude ${ }^{13} S_{\text {eff }} \approx \sqrt{D / \tau_{d}}$. Using this expression at threshold, where $\tau_{d} \approx 1 \mathrm{~ns}$ for both $6 \times 6$ and $12 \times 12 \mu \mathrm{m}^{2}$ oxide aperture devices with $I_{\text {th }} \sim 220$ and $600 \mu \mathrm{A}$, respectively, a value of $S_{\text {eff }} \sim 1 \times 10^{5} \mathrm{~cm} / \mathrm{s}$ was obtained. That it is in good agreement with previously reported values ${ }^{14}$ for strained InGaAs/ GaAs QW lasers, which where in the range of 1-2 $\times 10^{5} \mathrm{~cm} / \mathrm{s}$. Despite the relatively poor lateral carrier confinement, the estimated threshold carrier density of these oxide confined VCSELs is comparable to edge emitting lasers $^{2,11}$ and much lower than the estimated previously for implanted VCSELs. ${ }^{3}$ This would indicate that carrier spreading due to diffusion is not seriously limiting the operation of the VCSELs studied in this work. For very small devices, the values of $R_{m}$ and $R_{d}$ become very large at small currents. This fact sets a limitation on the technique applied here, since the $A, B, C$, and $D$ parameters cannot be accurately determined at very low bias currents. Hence, for very small devices, it would be necessary to solve the rate equation Eq. (2) including the diffusion term to get a more accurate estimation of the diffusion influence. ${ }^{15}$ However, for devices investigated here the simple approximations are still valid, leading to a consistent set of recombination parameters and diffusion coefficient.

In summary, differential carrier lifetimes of oxide confined VCSELs have been obtained directly from electrical impedance measurements by using a small-signal subthreshold equivalent circuit to model the measured laser impedance. The effects of lateral carrier diffusion on the characteristics of the devices have also been studied. Modeling of the experimental results indicates that carrier spreading due to diffusion is not seriously limiting the operation of the VCSELs studied here.

The authors want to thank G. E. Shtengel for very useful discussions, and M. Ochiai for her kindly assistance with sample characterization. Work at Texas Tech is supported by BMDO (monitored by Lou Lome), DARPA, and the J. F. Maddox Foundation.

${ }^{1}$ R. Olshansky, J. LaCourse, T. Chow, and W. Powazinik, Appl. Phys. Lett. 50, 310 (1987).

${ }^{2}$ G. E. Shtengel, D. A. Ackerman, and P. A. Morton, Electron. Lett. 31, 1747 (1995).

${ }^{3}$ G. E. Giudice, D. V. Kuksenkov, and H. Temkin, IEEE Photonics Technol. Lett. 10, 920 (1998).

${ }^{4}$ K. L. Lear, K. D. Choquette, R. P. Schneider, Jr., S. P. Kilcoyne, and K. M. Geib, Electron. Lett. 31, 208 (1995).

${ }^{5}$ J. Katz, S. Margalit, C. Harder, D. Wilt, and A. Yariv, IEEE J. Quantum Electron. QE-17, 4 (1981).

${ }^{6}$ R. S. Tucker, IEE Proc. I 128, 101 (1981).

${ }^{7}$ S. C. Kan and K. Y. Lau, IEEE Photonics Technol. Lett. 4, 528 (1992).

${ }^{8}$ S. Weisser, I. Esquivias, P. J. Tasker, J. D. Ralston, B. Romero, and J. Rosenzweig, IEEE Photonics Technol. Lett. 6, 1421 (1994).

${ }^{9}$ G. P. Agrawal and N. K. Dutta, Semiconductor Lasers, 2nd ed. (Van Nostrand Reinhold, New York, 1993), p. 37.

${ }^{10}$ M. Ochiai, Ph.D. dissertation, Colorado State University, 1997.

${ }^{11}$ M. S. Torre, I. Esquivias, B. Romero, K. Czotscher, S. Weisser, J. D. Ralston, E. Larkins, W. Benz, and J. Rosenzweig, J. Appl. Phys. 81, 6268 (1997).

${ }^{12}$ G. P. Agrawal and N. K. Dutta, Semiconductor Lasers, 2nd ed. (Van Nostrand Reinhold, New York, 1993), p. 125.

${ }^{13}$ P. D. Floyd, B. J. Thibeault, L. A. Coldren, and J. L. Merz, Electron. Lett. 32, 114 (1996).

${ }^{14}$ L. A. Coldren and S. W. Corzine, in Diode Lasers and Photonic Integrated Circuits, edited by K. Chang (Wiley-Interscience, New York, 1995), p. 150.

${ }^{15}$ B. J. Thibeault, T. A. Strand, T. Wipiejewski, M. G. Peters, D. B. Young, S. W. Corzine, and L. A. Coldren, J. Appl. Phys. 78, 5871 (1995). 